\title{
A Review Paper on Face Detection and Recognition in Video
}

\author{
Vishwanath D. Chavan ${ }^{1}$, Anil A Bharate ${ }^{2}$ \\ Dept. of CSE, Walchand Institute of Technology, Solapur, India ${ }^{1}$ \\ Department of Computer Science and Engineering, B.L.D.E.A's CET, Bijapur, India ${ }^{2}$
}

\begin{abstract}
The intention of this paper is to review various face detection and recognition methods, sort them into different categories and distinguish innovative trends. In this connection the face detection and recognition in video streams is the foremost significant step of information drawing out in many computer vision and image processing applications. Detecting and recognition of face in video stream in generally being a challenging problem, provides an enormous attention for recognition, classification, and activity analysis, making these later steps more efficient. Naturally, assumptions are made to constrain the detection and recognition problem in the perspective of a particular application. In this survey, investigation of many existing schemes in the literature of recent developments and general strategies of all these stages are done and limitations of the various methods and outline promising directions of research are discussed.
\end{abstract}

Keywords: Adaboost Algorithm, Haar Based Cascade classifier, Locality Preserving Projections.

\section{INTRODUCTION}

This paper is a sound towards developing a real time face recognition system which can recognize static images, which can be captured from video and can be modified to work with dynamic images. This is to improve the face detection system by using Haar Classifier to get higher accuracy result. Haar Classifier is used for face detection because it can detect the desire image very fast. The main challenge for a face recognition system is of effective feature extraction. In this paper, they offer a new linear dimensionality reduction algorithm, called Locality Preserving Projections (LPP). It forms a graph integrating neighbourhood information of the data set. They discussed concept of the Laplacian of the graph, then compute a transformation matrix which maps the data notions to a subspace. This linear transformation optimally conserves local neighbourhood information of the dataset.. This linear transformation optimally preserves local information in a certain sense.

The locality preserving projection is likely to be of particular use in information reclamation applications. If one wishes to retrieve video forms under a vector space model, then one will eventually need to do a nearest neighbour search in the low dimensional space. Since LPP is designed for preserving local structure, it is likely that a nearest neighbour search in the low dimensional space will yield similar results to that in the high dimensional space. This makes for an indexing scheme that would allow quick retrieval.

\section{THE REVIEWED APPROACHES ON FACE DETECTION}

A. S. Padmapriya (2012)

Padmapriya et al [1], as they discussed about face detection idea is pragmatic to real time car theft detection application. The face detection design is calculated using Adaboost algorithm in order to mine strong classifier using Haar classifiers. Every weak classifier supports a simple onset function on one of the features. Having such a large set of weak classifiers, AdaBoost knowledge is used to select a small number of weak classifiers and to combine them into a classifier deciding whether an image is a face or a non-face.

The face detection procedure bonds with haar features of human face. A simple feature is used in detection algorithm. In this paper the importance is given to the features than the numerical pixels because features can be used to represent both the statistically close facial information and lightly related background data sample image. The simplest form the features can be thought of as pixel intensity set estimations. This is where the sum of the luminance of the pixels in the white region of the feature is subtracted from the sum of the luminance in the remaining gray section.

The difference value is used as the feature value, and can be combined to form a weak supposition on regions of the image AdaBoost algorithm is to deal with very large collections of weak classifiers. In this paper, for face detection, very large training set has to be explored. In order to improve computational competence greatly and also reduce the false positive rate, a structure of gradually more complex classifiers called a cascade is built.

\section{B. Sarala A. Dabhade (2012)}

Sarala A. Dabhade et al [2], as they proposed the function of this module is to fix where in an image a face is located. The face detection entity works by scanning up an image at altered scales and looking for some simple patterns that denote the presence of a appears in the centre and presented at an even size. Face detection fixes where in an image a face is located. The face detection works by scanning up an image at different scales and looking for some simple forms that identify the presence of a face. 


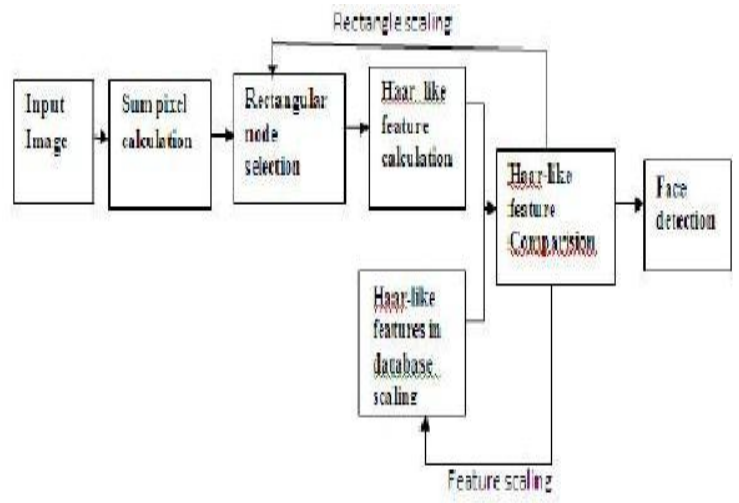

Fig. 1: Flow Diagram of the Face Detection.

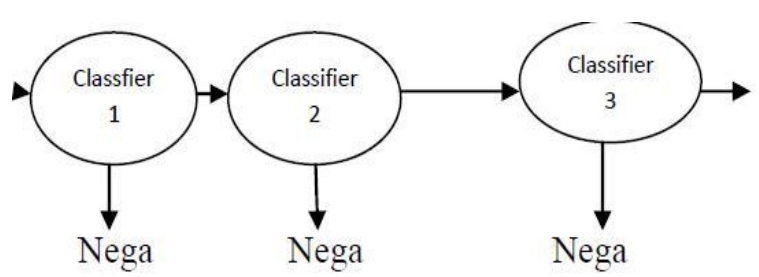

Fig. 2: The cascade classifier architecture.
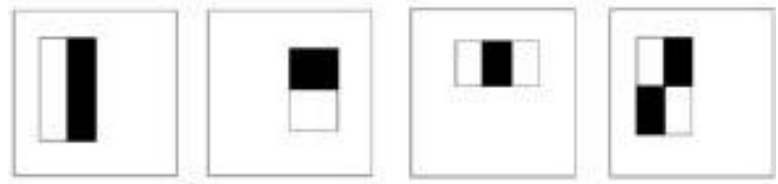

Fig. 3: Rectangle Feature of Haar-Like.

\section{Wen-Chang Cheng (2010)}

Wen-Chang Cheng et al [3], as they suggested a Cascade-Adaboost classifier, the architecture of which is shown in Fig. 2 where each classifier is Adaboost classifier Nega represents that they are determined to benegative examples by the input vector; Pis represents that they are determined to be positive examples by the input vector; $\mathrm{x}$ stands for input vector; if the input vector determines Adaboost classifier to be negative, the classifier will be removed from the training sample set and will not enter the next layer; so the number of samples reductions with the upturn of layers; in this way, negative samples are removed quickly; if it is determined to be positive, the training sample will enter the next layer to continue the classification until the last layer.

In this paper they have mentioned that because in cascade-Adaboost classifier, Adaboost classifier in visible layers could reach preset aims with less weak classifiers; but with the increase of layers, the samples of the remaining training set became less and similar; Adaboost classifier in rear layers need linear grouping of more weak classifiers to reach preset targets, which is easy to cause over fitting and time-consuming.

In this paper they uses rectangular features of Haar-like; as shown in Fig. 2 for feature extraction, it is an feature extraction method for image and object identification proposed by Viola and Jones it is an local block feature extraction method and is easy to extract; real time calculation can be finished by combining with the $f$ Integral image calculation method proposed by them; Four Haar-like secured rectangular features are used in this paper; protect the images of the candidate area above with the four protecting from up to down and from left to right; feature information can be achieved done by subtraction of sum of rectangle grey scale of black block and white block, and so forth. In addition, to notice pedestrians of different sizes, used different scales based on rectangle feature of each Haar-like and perform pedestrian detection to the candidate area with the cascade-Adaboost-SVM classifier in this paper.

\section{THE REVIEWED APPROCHES ON FACE RECOGNITION}

A. Quanquan $\mathrm{Gu}(2012)$

Quanquan et al [4], as they proposed an approach, called Locality Preserving Feature Learning (LPFL). LPFL aims to all together find a subset of features and a grouping of features, to optimize the Locality Preserving Criterion. LPFL take over the advantages of Laplacian score and LPI, as it is concurrently remove the unsuitable features and transform the suitable ones. The resulting optimization problem is a mixed integer programming problem, which is difficult to solve. Therefore, to relax it into a controlled Frobenius norm minimization problem and solved it using a variation of Alternating Direction Method. ADM iteratively updates the linear transformation matrix, the filtrate matrix and the Lagrangian multiplier. It is easy to implement and is theoretically guaranteed to converge.

The contributions of this paper are as follows:

(1) They proposed a new feature learning methodology based on Locality Preserving Principle, which is able to attain feature selection and transformation together.

(2) The number of particular features is overtly controlled in this method.

(3) They present a variation of ADM algorithm, which attains the overall solution of the proposed model with convergence rate $\mathrm{O}(1 / \mathrm{t})$ and

(4) Experimental results on benchmark document data sets showed that the proposed method outperforms LPI, Laplacian score and other state-of-the-art related approaches.

The rest of this paper is organized as follows. They reviewed on LPI and Laplacian score. They discussed about LPFL which includes feature selection into LPI. Experiments on benchmark document datasets are demonstrated. And also they reviewed on Locality Preserving Indexing and Laplacian Score. In this method also includes feature selection into Locality Preserving Indexing. The key idea is to find a subset of features, based on which they have learned.

\section{B. Shermina.J (2010)}

Shermija et al [5], as they discussed two standard algorithms, principal component analysis (PCA) and linear discriminant analysis (LDA), which are well-known for feature extraction and dimension reduction, widely used in face recognition. Both PCA and LDA effectively treasured only the linear multiple that based on the Euclidean structure. They fail to capture the fundamental structure 
which lies on a nonlinear sub manifold hidden in the image space. Recently, some nonlinear methods have been developed to discover the nonlinear structure of the manifold, algorithms e.g. Isomap, locally linear embedding (LLE), and Locality Preserving Projections (LPP) in which the first two algorithms are nonlinear but the LPP algorithm is a linear dimensionality reduction algorithm.

Locality Preserving Projections define face images by representing the face data onto a low-dimensional face feature subspace called "Laplacianfaces".

The advantages of LPP algorithm are that LPP is a linear method and it preserves the local information of the face image space. The limitation of LPP is that it represents an image by a vector in high-dimensional space which is often challenged with the difficulty that sometimes the image matrix is singular. It is unconfirmed and hence some of the important information with the classification are neglected. To overcome the complication of the singular problem, Laplacianface method projects the image set onto a PCA subspace to confirm to be non-singular.

In this paper, they projects the face data onto a Multilinear Principal Component Analysis (MPCA) subspace, and LPP algorithm is used to preserve the local structure information. By combining these two approaches considered as a global and local structure of the face image space can obtain a more effective optimal subspace for face representation and recognition. It follows two steps

1) First, it compresses and preserves the principal information in a matrix form, in order to remove more essential redundancy, and a much lower dimensional face representation is learned by which the recognition speed is enhanced.

2) Second, the low dimensional represent excepts the consequent LPP step from the singularity problem and it also achieves a more modest accurate recognition rate than the Laplacianface.

C. Veerabhadrappa (2010)

Veerbhadrappa et al [6], as they proposed a new dimensionality reduction technique called Diagona Locality Preserving Projections (DiaLPP) is proposed. Locality Preserving Projection (LPP) and Two Dimensional Locality Preserving Projection (2DLPP), DiaLPP directly pursues the ideal projection vectors from diagonal images without vector transformation. The 2DLPP method look for best projection vectors by using the row information of the image and the Alternate 2DLPP method try to find peak projection vectors by using the column information of the image, whereas the DiaLPP try for optimal projection vectors by connecting together the rows and column information of the images. Experimental results on subset of UMIST and ORL face database achieves higher recognition rate than 2DLPP, Alternate 2DLPP and DiaPCA (Diagonal Principal Component Analysis). The Kernel PCA finds principal components which are nonlinearly related to the input space by performing PCA in the space produced by the nonlinear mapping (through Kernels), where the low dimensional suppressed structure is easier to discover. Both Isomap and LLE preserves the local neighborhood features while trying to obtain highly non linear embeddings. But LLE and Isomap fail when data are complex and noisy. So, S-Isomap is proposed to recover the true manifold of the noisy data and to preserve the class label information.

Locality Preserving Projection (LPP) finds an embedding that preserves local information and obtain face subspace which detects the essential face manifold structure. If training samples are not enough and data dimension is high, especially for image data LPP can ${ }^{\text {ee }} t$ be used directly due to singularity of matrices it estimates the image covariance matrix more accurately and computes the corresponding eigenvectors more efficiently than PCA and these methods operates on image matrix and works in row direction of the image.

The 2DPCA and 2DLPP reflects only the information between rows, which results column information is missing. Similarly, Alternate 2DPCA and Alternate 2DLPP reflects only column information by neglecting the row information. This implies that with standard 2DLPP or Alternate 2DLPP method, we can only capture either row or column information at the same instant. We attempt to solve this problem by transforming the original faces into corresponding diagonal face images. Because the rows (or columns) in the transformed diagonal images simultaneously integrate the information of rows and columns in the original images, it can reflect both information between rows and columns.

Suppose that there are $\mathrm{M}$ images denoted by $\mathrm{m}$ by $\mathrm{n}$ matrices Ak $(\mathrm{k}=1,2 \ldots \mathrm{M})$. For each image, define the corresponding diagonal image as follows:

(1) If the height $\mathrm{m}$ is equal to or smaller than the width $\mathrm{n}$, use the method illustrated in Figure 1 to generate the diagonal image $\mathrm{D}$ for the original image $\mathrm{A}$.

(2) If the height $m$ is bigger than the width $n$, use the method to generate the diagonal image $\mathrm{D}$ for the original image $\mathrm{A}$.

(3) After obtaining the diagonal images for all the M images, apply the 2DLPP algorithm.

\section{CONCLUSION}

A face detection method based on cost-sensitive AdaBoost is presented in the paper. Improved AdaBoost algorithm achieves more robust performance and higher speed over conventional AdaBoost-based methods. Comparative results on test sets demonstrate the effectiveness of the algorithm. In this article, the importance of Locality Preserving Projection is studied and the comparison of existing face recognition technique with the impact of LPP is also analyzed.

\section{REFERENCES}

[1] S.Padmapriya \& Esther Annlin KalaJames „Real Time Smart Car Lock Security System Using Face Detection and Recognition ${ }^{\text {ee }}, 2012$ International Conference on Computer Communication and 
Informatics (ICCCI - 2012), Jan. 10 - 12, 2012, Coimbatore, INDIA.

[2] Sarala A. Dabhade \& Prof. Mrunal S. Bewoor „Real Time Face Detection and Recognition using Haar - Based Cascade Classifier and Principal Component Analysis" International Journal of Computer Science and Management Research Vol 1 Issue 1 Aug 2012 ISSN: 2278-733X.

[3] Wen-Chang Cheng and Ding-Mao Jhan, „A Cascade Classifier Using Adaboost Algorithm and Support Vector Machine for Pedestrian Detection" IEEE trans. on Pattern Analysis and Machine Intelligence, Vol. 32, No. 7, pp. 1239 - 1258, 2010

[4] Quanquan Gu Marina Danilevsky Zhenhui Li Jiawei Han, Locality Preserving Feature Learning "e 15th International Conference on Artificial Intelligence and Statistics (AISTATS) 2012, La Palma, Canary Islands. Volume XX of JMLR: W\&CP XX.

[5] Shermina.J, „Application of Locality Preserving Projections in Face Recognition", (IJACSA) International Journal of Advanced Computer Science and Applications, Vol. 1, No. 3, September 2010

[6] Shou-Jen Lin, Chao-Yang Lee, Mei-hsuan Chao, Chi-Sen Chiou, Chu-Sing Yang, ,,The Study And Implementation Of Real-Time Face Recognition And Tracking System"e, Proceedings of the Ninth International Conference on Machine Learning and Cybernetics, Qingdao, 11-14 July 2010.

[7] Łukasz Stasiak, Andrzej Pacut, Raul Vincente-Garcia," Face Tracking and Recognition in Low Quality Video Sequences with the Use of Particle Filtering"e.

[8] Veerabhadrappa and Lalitha Rangarajan," Diagonal Locality Preserving Projection as dimensionality Reduction Technique with Application to Face Recognition", IJCA Special Issue on "Recent Trends in Image Processing and Pattern Recognition" RTIPPR, 2010

[9] Yasir M. Mustafah, Abbas Bigdeli, Amelia W. Azman and Brian C. Lovell,“ Face Detection System Design for Real Time High Resolution Smart Camera",2009 IEEE.

[10] Jiwen Lu, Yap-PengTan, "Improved discriminant locality preserving projections for face and palmprint recognition ${ }^{\text {ee }}, 2011$ Elsevier. Our work is to implement the face detection and recognition and in smart car lock security system. Xiaohu Maa, Yanqi Tan, Yaying Zhao, Hongbo Tian, " Face Recognition Based on Maximizing Margin and Discriminant Locality Preserving Projection", Journal of Information \& Computational Science,2010 IEEE. Information \& Computational Science, 2010 IEEE.

[11] Haitao Zhao and Shaoyuan Sun, "OPTIMAL LOCALITY PRESERVING PROJECTION"e, Proceedings of 2010 IEEE 17th International Conference on Image Processing September 26-29, 2010, Hong Kong.

[12] Minghua WAN, Zhong JIN, Zhihui LAI, Jun YIN, “" Feature Extraction and Classification Using Fuzzy Linear Preserving Projections (FLPP)"e, Journal of Computational Information Systems6:5(2010) 1475-1482. 